Mid-Infrared Sensor for Hydrocarbon Monitoring: The Influence of Salinity, Matrix and Aging on HydrocarbonPolymer Partitioning

\begin{tabular}{|r|l|}
\hline Journal: & Analytical Methods \\
\hline Manuscript ID & AY-ART-12-2017-002874.R1 \\
\hline Article Type: & Paper \\
\hline Date Submitted by the Author: & 14-Feb-2018 \\
\hline Complete List of Authors: & $\begin{array}{l}\text { Stach, Robert; Institut für Analytische und Bioanalytische Chemie, } \\
\text { University of Ulm } \\
\text { Pejcic, Bobby; CSIRO, Earth Science and Resource Engineering } \\
\text { Heath, Charles; CSIRO, Earth Science and Resource Engineering } \\
\text { Myers, Matthew; CSIRO, Environmental Science and Resource Engineering } \\
\text { Mizaikoff, Boris; Institut für Analytische und Bioanalytische Chemie, } \\
\text { University of Ulm }\end{array}$ \\
\hline \hline
\end{tabular}




\title{
Mid-Infrared Sensor for Hydrocarbon Monitoring: The Influence of Salinity, Matrix and Aging on Hydrocarbon-Polymer Partitioning
}

\author{
Robert Stach $^{\mathrm{a}, \mathrm{b}}$, Bobby Pejcic $^{\mathrm{a} *}$, Charles Heath $^{\mathrm{a}}$, Matthew Myers $^{\mathrm{a}}$, Boris Mizaikoff $^{\mathrm{b}}$ \\ ${ }^{a}$ CSIRO, Energy, 26 Dick Perry Ave, Kensington, WA, 6151, Australia. \\ ${ }^{\mathrm{b}}$ Ulm University, Institute of Analytical and Bioanalytical Chemistry, Albert-Einstein-Allee \\ 11, 89081 Ulm, Germany.
}

*Corresponding author: E-mail: Bobby.Pejcic@csiro.au Tel.: +61-8-6436-8814. 


\section{Abstract}

Mid-infrared sensors (MIR) based on attenuated total reflection (ATR) spectroscopy provide a robust and sensitive platform for the detection of low levels of organic molecules dissolved in water. The chemical and partitioning properties of the polymer membrane play an important role and are crucial to MIR-ATR sensor performance. Although the MIR-ATR sensor has been used to quantify dissolved hydrocarbons in a wide range of aquatic environments, very little is reported on the impact of aqueous solution composition and polymer membrane aging on the hydrocarbon-polymer partitioning process. In the present study we investigated a number of factors that may affect the sensor sensitivity and analytical performance during quantification of hydrocarbons in water. Calibration curves were obtained as a function of salinity and it was revealed that the response factors are generally higher in the presence of salts. We show that reliable hydrocarbon analysis can be achieved in water provided that the sensor is calibrated using appropriate standard solutions and corrected for salinity/ionic strength variations. Sensor measurements were also performed over a range of crude oil-water mixtures, and these studies revealed that the total petroleum hydrocarbon concentration in solution (between the 0-20 ppm range) has a negligible effect on the analyte-polymer partitioning process. In terms of sensor durability, the polymer film displayed remarkable repeatability and long-term stability in water over a two-month testing period.

Keywords: hydrocarbon; optical sensor; mid-infrared spectroscopy; attenuated total reflectance; polymer membrane; water quality; environmental monitoring. 


\section{Introduction}

Over the last several decades there has been considerable interest in understanding the environmental, economic and social impact of organic contaminants that enter aquatic systems. ${ }^{1,2}$ Monitoring the type and amount of organic-based toxins is an important aspect of ensuring the quality and safety of water supplies. ${ }^{3}$ Although many different analytical techniques are available for detecting hydrocarbons in water, ${ }^{4}$ there still is a need for improved water monitoring systems which are able to provide real-time information that leads to a faster operational response during a pollution event. Chemical sensors and portable onsite instrumentation that are capable of delivering rapid and direct information on organic contaminants without any significant sample pre-treatment have been developed. ${ }^{5}$ Despite achieving sensitivities which are comparable to standard laboratory methods, satisfactory selectivity and molecular recognition still remain a significant challenge when analysing real environmental samples.

The sensor based on mid-infrared (MIR) attenuated total reflectance (ATR) spectroscopy is a well-established method for detecting and discriminating many different types of hydrocarbons. ${ }^{6-9}$ An important component of the sensor is the polymer membrane that is coated onto an MIR transparent internal reflection element based on ATR crystals or optical fibers. ${ }^{10}$ The analytes are extracted from solution into the film and the molecules gradually diffuse to the region of the evanescent field where they interact with the MIR radiation. A hydrophobic polymer layer is normally used and this is responsible for: 1) allowing hydrophobic/non-polar molecules and/or molecules of low polarity to be enriched in the polymer film; 2) minimising the sorption/diffusion of water and other unwanted interfering molecules from the evanescent field; and 3) helping protect the ATR crystal/optical fiber from physical and chemical stress. The type of polymer and its film thickness are important factors 
that need to be considered when designing a MIR-ATR sensor for hydrocarbons. ${ }^{11}$ Although there are many different types of polymers, only a few appear to be suitable for detecting low molecular weight hydrocarbons in water. Ethylene/propylene copolymer, ${ }^{12}$ poly(acrylonitrileco-butadiene), ${ }^{13}$ poly(styrene-co-butadiene), ${ }^{13}$ polydimethylsiloxane, ${ }^{13}$ polyisobutylene,${ }^{14-16}$ poly(vinyl chloride), ${ }^{17,18}$ Teflon, ${ }^{19}$ polystyrene, ${ }^{20}$ and poly(methyl methacrylate) ${ }^{21}$ are some of the polymers that have been investigated for extracting aromatic-based hydrocarbons. Various reports have been published comparing the partitioning and diffusion properties of different polymers $^{13,22,23}$ and polymers that exhibit the greatest hydrocarbon extraction/enrichment and diffusion rates are generally ones that are amorphous and rubbery. Other studies have shown that the sensitivity and analytical performance of the MIR-ATR sensor can be significantly improved when the polymers incorporate other materials/substances such as plasticisers, ${ }^{17}$ functionalized carbon nanotubes ${ }^{24,25}$ and calixarenes. ${ }^{21,26}$

Recent work revealed that the MIR-ATR sensor is able to identify and quantify a range of aromatic hydrocarbons in pure ${ }^{16,27}$ and saline water. ${ }^{28}$ Although reasonable agreement was obtained when compared to the standard chromatographic method, it was observed that the concentration of certain hydrocarbon analytes are over predicted ( $>50 \%$ difference) suggesting the possibility of interference effects on the sensor response. In addition, it was found that the quantification of xylenes is more problematic in seawater which had been exposed to diesel compared to seawater containing dissolved crude oil. ${ }^{28}$ Previous reports also found that the concentration measured by the sensor for other volatile organic compounds in groundwater was higher compared to the reference method. ${ }^{29}$ Other researchers revealed that the amount of natural organic matter present in water has a profound effect on the sensor response. ${ }^{30}$ Considering that the response mechanism of the MIR-ATR sensor is governed by hydrocarbon molecules partitioning into a polymer film it is possible that the hydrocarbon- 
polymer extraction process is influenced by the solution properties and composition. A number of studies have shown that the water-polymer partition coefficients of hydrocarbons and hydrophobic organic compounds depends on various factors such as the solvent composition, ${ }^{31,32}$ salinity, ${ }^{33}$ and temperature. ${ }^{33}$

Although the MIR-ATR sensor has been extensively used to quantify dissolved hydrocarbons in a wide range of aquatic environments (freshwater, groundwater, seawater), very little is reported on the impact of aqueous solution properties and matrix composition (i.e., ionic strength, hydrocarbon type and concentration) on the sensor response. In addition, the influence of chemical interferences and polymer membrane aging has not been studied in any great detail and further research is needed to address this before the MIR-ATR sensor can be routinely deployed for the analysis of hydrocarbon compounds in natural waters. The objective of this report is to determine and understand the factors that affect the analytical performance of the MIR-ATR sensor during calibration and quantitative measurement of hydrocarbons in real natural waters.

\section{Experimental}

\subsection{Materials and reagents}

Toluene (99.8\%), $m$-xylene (99\%), $p$-xylene (99\%) and naphthalene (99\%) were all obtained from Sigma-Aldrich and used without further purification. Polyisobutylene (PIB) was supplied by Scientific Polymer Products, Inc. (Ontario, NY, USA) and used as provided. Methanol and n-hexane were obtained from Merck (Darmstadt, Germany) as a Cromasol purity grade. All organic solvents were AR grade and used without further purification. The crude oil was obtained from North West Shelf, Australia. The saline water was prepared using the composition reported elsewhere. ${ }^{34}$ 


\subsection{Diamond surface preparation and coating deposition}

Diamond (area of $2 \mathrm{~mm} \times 2 \mathrm{~mm}$ ) was used as the internal reflection element (IRE) for ATR measurements (provides a single reflection). Prior to polymer (i.e. PIB) film deposition, the surface of the diamond was cleaned by wiping with acetone-wet lens-cleaning tissue (Whatman International Ltd., Maidstone, England) followed by rinsing with acetone and drying by flowing high purity nitrogen gas for several minutes. A PIB coating was prepared by dissolving the polymer $(1.0 \% \mathrm{w} / \mathrm{w})$ in $\mathrm{n}$-hexane and sonication for about $1 \mathrm{hr}$ followed by drop casting onto the IRE. The polymer-coated diamond was allowed to dry overnight (film thickness of approx. $10 \mu \mathrm{m}$ ) and IR spectra were collected to confirm that the solvent had completely evaporated. To allow comparison between measurements, the same coating was used for the repeat measurements.

\subsection{Instrumentation and sensing system}

All ATR measurements were performed using a Bruker Alpha Fourier transform infrared spectrometer (FTIR) equipped with a deuterated 1-alanine triglycine sulfate (DLATGS) detector. A similar experimental setup was used to that reported elsewhere. ${ }^{28}$ A stainless steel flow cell was mounted onto the ATR unit and all aqueous solutions were pumped across the polymer-coated diamond surface using a peristaltic pump (Ismatec, IDEX Corporation). Prior to analytical measurements, the polymer coating was equilibrated in deionized water (MilliQ water) for a period of $24 \mathrm{hrs}$. All experiments were performed at room temperature and data were recorded in the spectral range between $4000-400 \mathrm{~cm}^{-1}$ at a spectral resolution of $2 \mathrm{~cm}^{-1}$. The number of scans was varied depending on the nature of the experiment. The wavelengths used during the sensor measurement of toluene, $m$-xylene, $p$-xylene and naphthalene are as follows $727 \mathrm{~cm}^{-1}, 767 \mathrm{~cm}^{-1}, 794 \mathrm{~cm}^{-1}$, and $781 \mathrm{~cm}^{-1}$, respectively. 


\subsection{Sensor calibration and measurements in water}

A multianalyte stock solution ( $2000 \mathrm{ppm})$ was prepared by dissolving toluene, $m$-xylene, $p$ xylene and naphthalene in pure methanol. Calibration curves were established in the concentration range between 0.5 to $10 \mathrm{ppm}$ by serial dilution of the multianalyte stock solution in either deionised or saline water $(3.5 \%$ salinity) containing methanol $(0.5 \% \mathrm{v} / \mathrm{v})$. The concentration of the naphthalene standards varied from 50 to $1000 \mathrm{ppb}$. Methanol was added to help improve the stability and solubility of the hydrocarbons in water and previous studies have shown that methanol does not interfere with the sensor measurements. ${ }^{12}$ All analytical calibration curves were generated using at least four standard solutions and the sensor calibration was independently repeated at least two times. To limit evaporation losses due to the volatile nature of the hydrocarbons, all standards were freshly prepared and all subsequent studies were performed within less than 4 hours. Only glass with minimal headspace was used throughout these studies to prepare and store the solutions.

\subsection{Spectral data analysis}

The characteristic infrared absorption features were evaluated via peak area analysis using the software package OPUS (Bruker Optics, Ettlingen, Germany). The regions integrated are based on the procedure described elsewhere. ${ }^{28}$ For all spectra the polymer coated diamond in deionized water was used as a background. At least duplicate measurements were performed and the peak areas from each spectral region were averaged.

\subsection{Effect of total petroleum hydrocarbons (TPH)}

Studies were also undertaken to investigate the effect of dissolved oil concentration on the sensor response. The total petroleum hydrocarbons (TPH) in water was prepared according to a procedure outlined elsewhere. ${ }^{28,35}$ Briefly, different amounts of crude oil were placed in 
separate glass vessels that contained a similar volume of deionised water and these were allowed to equilibrate for a period of 3 days. The hydrocarbon concentrations and TPH in the aqueous solutions were verified using gas chromatography (GC) and gas chromatographymass spectrometry (GC-MS).

\subsection{Validation using gas chromatography}

The toluene and xylene compounds were determined using a HP 6890 GC MSD with an EST Encon purge-and-trap concentrator via a HP 624 (25 m x $0.2 \mathrm{~mm}$ ID) column. Helium was bubbled through the water sample, and the vapour was swept through a sorbent trap (Vocarb 3000). The column conditions were: split ratio of 50 to 1 ; initial column temperature of $40^{\circ} \mathrm{C}$; final column temperature of $245^{\circ} \mathrm{C}$; column flow of $1.0 \mathrm{ml} / \mathrm{min}$ in $\mathrm{He}$; and heating rate of $14^{\circ} \mathrm{C} / \mathrm{min}$. Naphthalene was liquid/liquid extracted with dichloromethane and analysed via an Agilent 6890 GC / Agilent 5973 inert MSD. A J\&W DB-5MS (30 m x 0.25 mm x 0.5 mm) column was used for naphthalene analysis and the conditions used are as follows: splitless mode; $300^{\circ} \mathrm{C}$ injector temperature; $1 \mu \mathrm{l}$ injector volume.

\section{Results and discussion}

\subsection{Sensor response and reversibility}

Some of the factors that influence the polyisobutylene film sensitivity was previously investigated by $\mathrm{us}^{28}$ and others ${ }^{36}$ and is not the aim here to repeat that work. To evaluate the suitability of the MIR-ATR sensor for real-time measurements and analysis, a study was performed by exposing the sensor to alternate solutions in the presence and absence of hydrocarbons. Figure 1 shows a typical transient response when subjected to various concentrations (50 and $100 \mathrm{ppm}$ ) of $p$-xylene in deionised water. It is evident that the absorbance signal arising from the polymer extraction of $p$-xylene is proportional to 
concentration and this is in agreement with the combined Beer-Lambert law and solid-phase microextraction theory which is described elsewhere. ${ }^{37}$ In terms of the response time, equilibrium was reached relatively rapidly ( $<5 \mathrm{mins})$ compared to previous studies that used a similar polymer film. ${ }^{16}$ Notwithstanding, the PIB layer utilised in the present study was slightly less thick, and is consistent with $p$-xylene taking a shorter time to diffuse through the polymer and into the region of the evanescent wave.

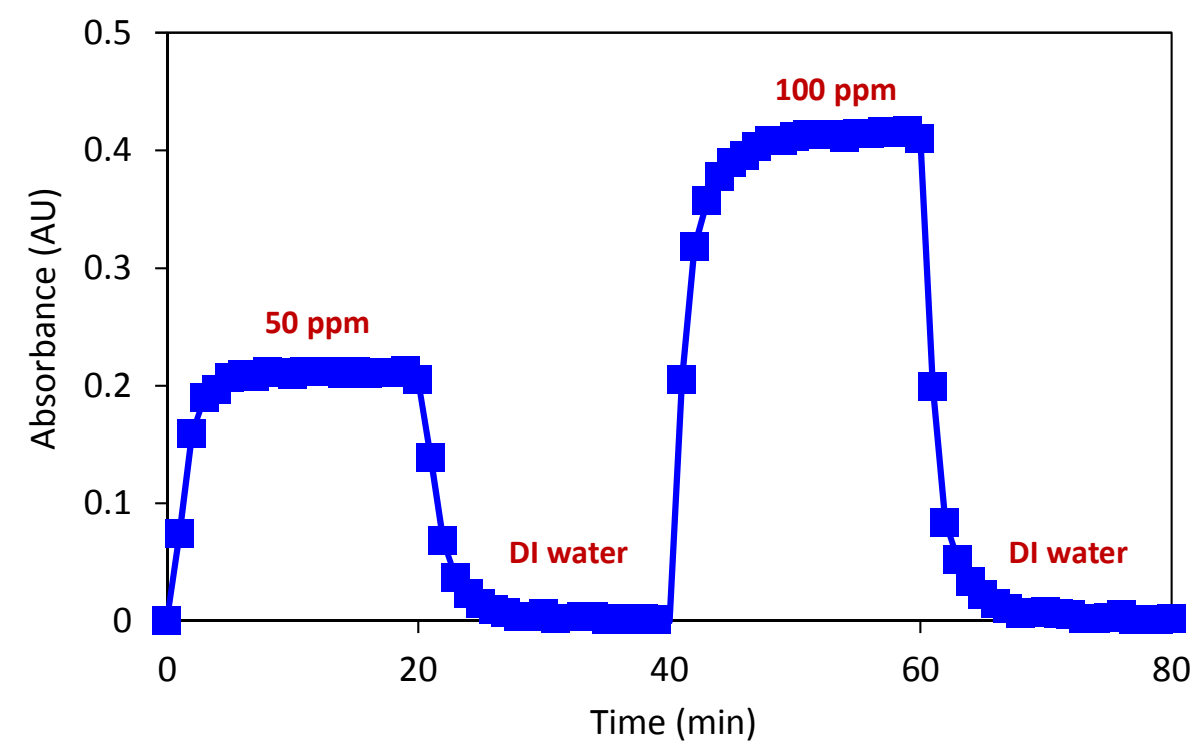

Figure 1. The transient response of the MIR-ATR sensor when exposed to an aqueous solution comprising different $p$-xylene concentrations. The IR spectra were collected using 24 scans at $2 \mathrm{~cm}^{-1}$ resolution and $4 \mathrm{~mL} \mathrm{~min}^{-1}$ flow rate.

To investigate if the response time varied as a function of hydrocarbon concentration, the period taken to achieve $90 \%\left(\mathrm{~T}_{90}\right)$ of the maximum absorbance value was determined. The time taken to reach a steady state signal varied slightly and the $\mathrm{T}_{90}$ was a little lower for a PIB film exposed to $50 \mathrm{ppm}(3 \pm 0.5 \mathrm{~min})$ compared to the $100 \mathrm{ppm}(4 \pm 0.5 \mathrm{~min}) \mathrm{p}$-xylene solution. Previous studies revealed that the hydrocarbon analyte diffusion coefficient through a polymer film varies with the hydrocarbon concentration ${ }^{38}$ and that the slight response time difference may be attributed to either diffusion coefficient variations or variations in the film 
properties (i.e., polymer swelling). The $\mathrm{T}_{90}$-time was determined for other hydrocarbon analytes and the response time generally increased in the following order: toluene $<$ o-xylene $=$ p-xylene $<$ naphthalene. Generally, other aspects being equal, the diffusion for the smaller molecules will be faster while the diffusion for larger molecules will be slower and the order of response times is consistent with the molecular size of each of the analytes. This study confirms that the response time for a PIB-coated diamond-based (single reflection) MIR-ATR sensor depends on both the hydrocarbon type and concentration and is consistent with other previous reports that used a multi-reflection ATR. ${ }^{13,16}$

Sensor reversibility/recovery was also evaluated and it is evident from Figure 1 that a certain period of time was required for the sensor to reach a baseline value after exposure to pure water. The time taken to completely remove the $p$-xylene $(50 \mathrm{ppm})$ from the polymer film and achieve an almost zero absorbance reading varied between 8 to 10 mins for our experimental conditions. Interestingly, complete membrane recovery was similar $(9 \pm 1$ mins $)$ when the sensor was exposed to the higher $p$-xylene concentration (i.e., $100 \mathrm{ppm}$ ). Although the hydrocarbon concentration used in this study is much higher than that typically encountered in actual aquatic environments, further research is still needed to reduce the sensor response time if real-time measurements are to be satisfactorily achieved. A number of methods have been used (i.e., heating, solvent washing, air purging) to increase the diffusion and removal of analyte molecules from the polymer membrane. Recent work has shown that the diffusion properties of PIB can be substantially improved when it is chemically modified with a plasticiser molecule. ${ }^{39}$ It was revealed that the free volume size and distribution of the PIB film increases considerably after the introduction of a plasticiser and this presumably may lead to faster response and recovery times. Although the sensor design and geometry have not been optimised here given that a commercial system was used, it is well known that the flow 
cell configuration has a significant effect on the sensing performance. ${ }^{40}$ Irrespective, this study suggests that both the response and recovery times need to be considered if reliable and accurate sensor measurements are to be performed on natural water samples.

\subsection{Effect of water aging}

Although the polymer coated MIR-ATR sensor has been widely used to detect hydrocarbons in aqueous solutions, very little information exists regarding its stability and suitability over extended periods in water. To investigate its long-term analytical performance, the MIR-ATR sensor was continuously exposed to a number of different solutions (i.e., deionised water, seawater, oil-water mixtures) at various stages noting that the same PIB film was used throughout the entire study. Regular calibrations and checks of the sensor were made in standard hydrocarbon solutions and the results obtained are shown in Figure 2. The absorbance of a freshly prepared $p$-xylene solution varied between $0.039 \pm 0.004$ AU $(1 \sigma)$ over the 65 day period which represents a variation of just over $10 \%$. Evidently the response to the $p$-xylene standard solution did not deviate considerably with time suggesting that longterm exposure to various aqueous solutions does not pose any major problem for the polymer membrane. It is worth noting that no sensor checks/calibrations were performed in the $p$ xylene solution between 10 to 35 days as the polymer film was continuously exposed in water for that entire period. IR spectra were also periodically collected and this did not reveal any significant chemical changes in the PIB film (not shown). These results are consistent with previous studies which show that polymer films with a low glass transition temperature are less susceptible to hydrocarbon sensitivity changes due to water aging effects compared to polymers that have a high glass transition temperature. ${ }^{41}$ In the case of our PIB-based MIRATR sensor, the slight variations observed in sensitivity are most likely attributable to daily temperature fluctuations $\left(20 \pm 2^{\circ} \mathrm{C}\right)$ in the laboratory along with deviations in the $p$-xylene 
concentration $(9 \pm 1 \mathrm{ppm})$ rather than any degradation of the polymer film/sensing surface. In fact, recent work by us has revealed that high temperatures have a much greater effect on both the polymer enrichment/sensitivity for hydrocarbons and film degradation. ${ }^{42}$ Regardless, this study shows that the PIB-coated diamond ATR does not undergo any significant deterioration (at room temperature) in response over a two month period and that the sensor may be suitable for long-term monitoring studies in aquatic environments.

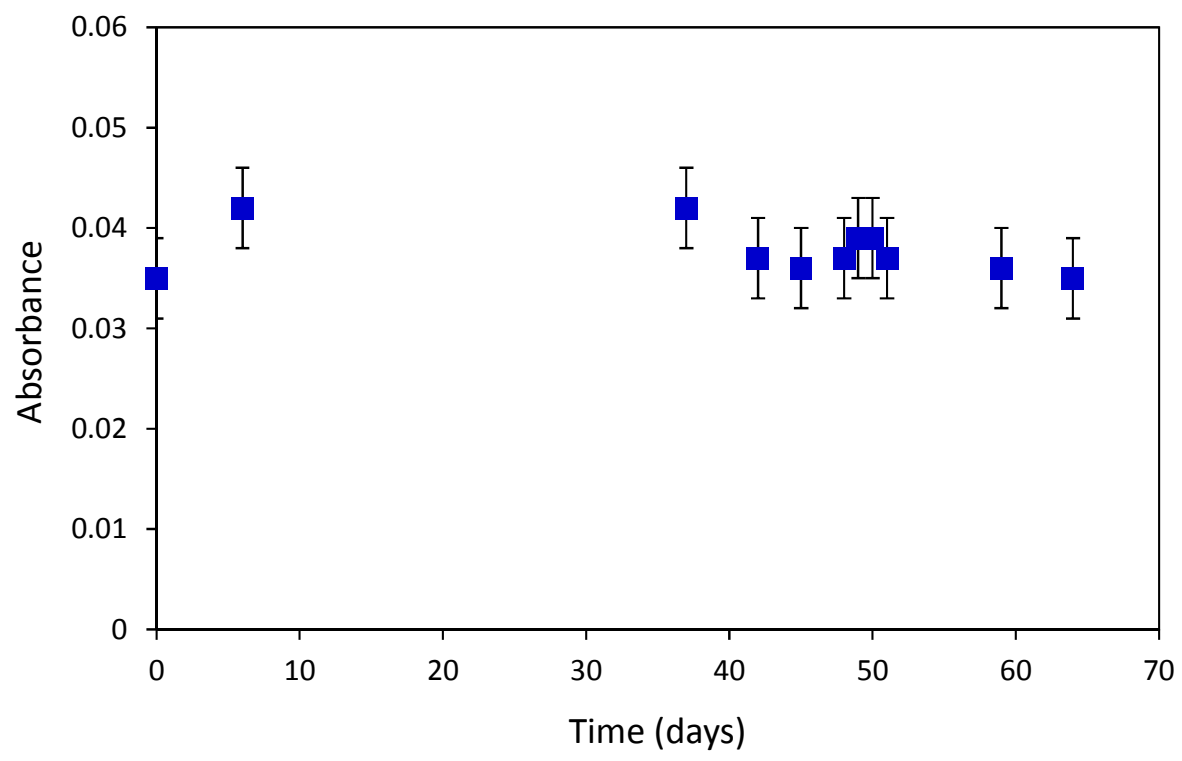

Figure 2. Evaluation of sensor stability as a function of time. Readings were obtained by exposing the sensor periodically to a $10 \mathrm{ppm} p$-xylene standard. The error bars represent the standard deviation of the eleven replicate measurements.

\subsection{Effect of salinity}

Previous reports found that the response of an ethylene-propylene copolymer coated MIRATR sensor was unaffected by variations in salinity during the determination of chlorinated hydrocarbons in marine water. ${ }^{43}$ However, recent work using a similar polymer film revealed significant intensity differences in the IR spectra of various aromatic hydrocarbons when exposed to seawater comprising a dissolved oil mixture. ${ }^{27}$ To investigate the effect of solution composition on the sensor response a study was performed to determine the calibration 
functions in pure water versus saline water. Figure 3 shows the calibration curves of the respective hydrocarbons (i.e., toluene, $m$-xylene, $p$-xylene and naphthalene) and it is evident that significant differences were observed. The response was generally larger in saline relative to deionised water and is most likely related to variations in the hydrocarbon-polymer enrichment process. Other researchers also observed a higher hydrocarbon absorbance signal with increased salinity and attributed this to a reduction in the hydrocarbon solubility in water along with a more favourable polymer enrichment process. ${ }^{44,45}$ Interestingly, at low toluene, $m$-xylene, $p$-xylene (below $2 \mathrm{ppm}$ ) and naphthalene (below $0.2 \mathrm{ppm}$ ) concentrations the difference in the absorbance intensity was negligible and is in agreement with observations made elsewhere which found that salinity has no effect on the sensor response. ${ }^{43}$ However, at higher hydrocarbon concentrations the absorbance variations between saline and deionised waters were much greater (see Figure 3), suggesting that salinity may have an effect on the MIR-ATR sensor response.
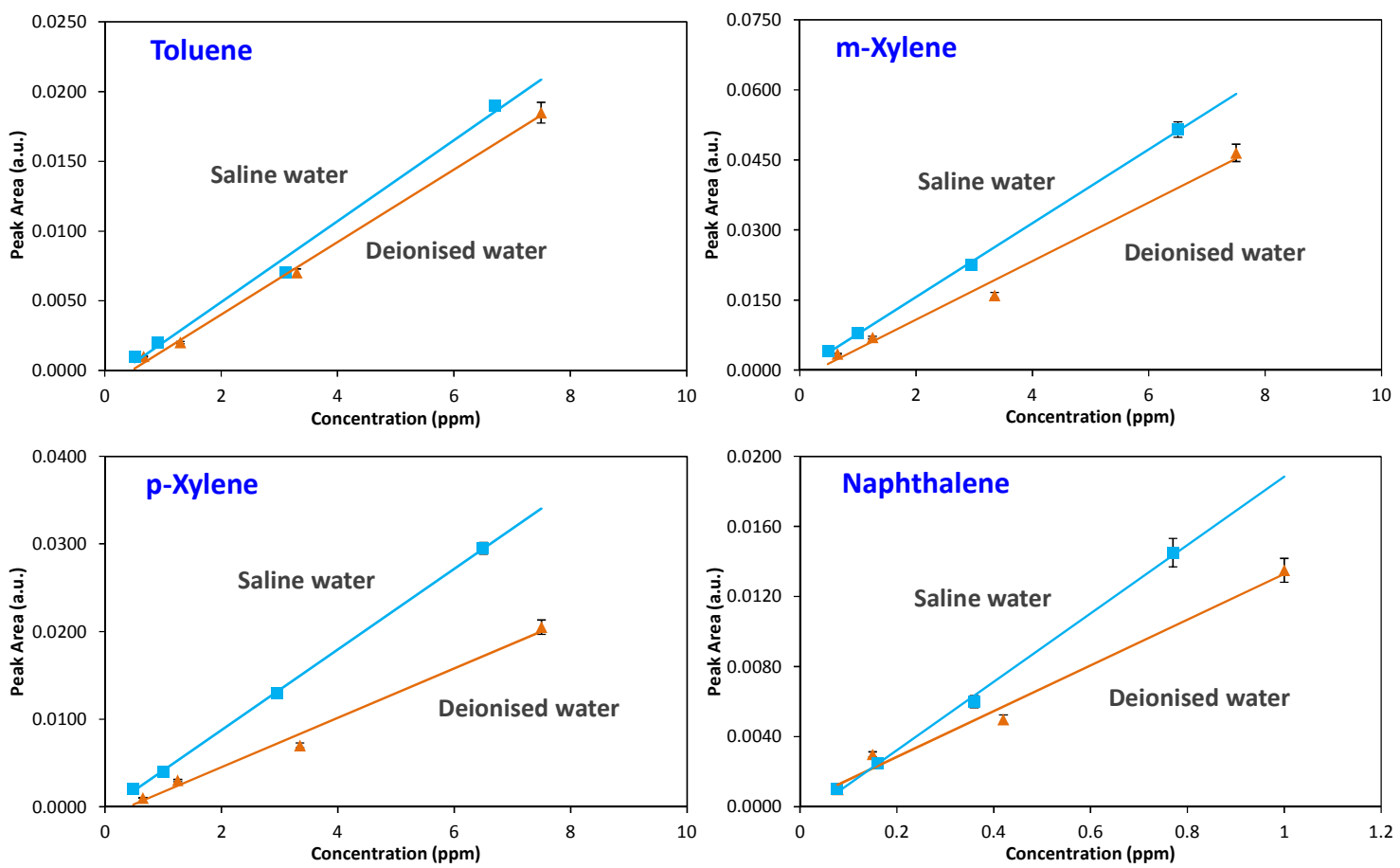

Figure 3. Calibration curves of the MIR-ATR sensor in various hydrocarbon solutions in the presence (salinity $=3.5 \%$ ) and absence of inorganic salts. 
Linear regression was performed on the hydrocarbon analytes (i.e., toluene, $p$-xylene, $m$ xylene and naphthalene) as a function of concentration and Table 1 summarises the response factors and correlations. It is evident that a linear response was obtained in deionised water over the concentration range 0.5 to $8 \mathrm{ppm}$ for toluene, $m$-xylene, and $p$-xylene, whereas for naphthalene the response was linear between 0.05 to $1 \mathrm{ppm}$. The sensitivities/response factors are comparable and agree favourably with previous publications that use a similar polymer film. ${ }^{28}$ A linear response was also achieved in saline water over a similar concentration range; however, the slope of the calibration curve was notably higher compared to deionised water. The larger slope for saline compared to deionised water is due to enhanced partitioning and this is consistent with recent studies that show variations in the polymer-water partition coefficient as a function of salinity. ${ }^{33}$ Jonker et al. (2015) found that the polymer-water partition coefficient of low molecular weight polycyclic aromatic hydrocarbons generally increases with increasing salinity. ${ }^{33}$ In addition, gas chromatography measurements on the various aqueous solutions confirmed that the hydrocarbon solubility was slightly lower in saline compared to deionised water when the saturated hydrocarbon solutions were preparing under similar conditions (i.e., same temperature).

Table 1. Calibration functions derived from the MIR-ATR sensor in various aqueous solutions. Note that calibration standards were prepared in the range between 0.5 to $7 \mathrm{ppm}$ for toluene, $m$-xylene and $p$-xylene, whereas for naphthalene the region between 0.07 to $1 \mathrm{ppm}$ was used.

\begin{tabular}{|c|c|c|c|c|}
\hline & \multicolumn{2}{|c|}{ Deionised water } & \multicolumn{2}{c|}{ Saline water } \\
\hline Molecule & Linear fit (a.u./ppm) & $\boldsymbol{R}^{\mathbf{2}}$ & Linear fit (a.u./ppm) & $\boldsymbol{R}^{\mathbf{2}}$ \\
\hline Toluene & $y=0.0026 x-0.0012$ & 0.9978 & $y=0.0029 x-0.0009$ & 0.9919 \\
\hline $\boldsymbol{m}$-Xylene & $y=0.0063 x-0.0017$ & 0.9875 & $y=0.0079 x-0.000085$ & 0.9995 \\
\hline $\boldsymbol{p}$-Xylene & $y=0.0028 x-0.0011$ & 0.9895 & $y=0.0046 x-0.0004$ & 0.9998 \\
\hline Naphthalene & $y=0.0130 x+0.0003$ & 0.986 & $y=0.0195 x-0.0007$ & 0.9984 \\
\hline
\end{tabular}


A closer look at the data revealed that the slopes for the $p$-xylene and naphthalene calibrations varied by more than $50 \%$ whereas the $m$-xylene and toluene variations were much less (11 to $25 \%$ ). Interestingly the slopes did not change by a similar amount and is probably related to non-linear variations in either the hydrocarbon-water solubility and/or hydrocarbon-polymer partition coefficient with salinity. It is important to note that the y-intercepts obtained in the linear regression are all very near the origin as would be expected for response to no analyte. Nevertheless, the differences in the response slope confirms that the hydrocarbon-polymer partition process is dependent on salinity and this is the reason for the variations observed in MIR-ATR sensor sensitivity. Furthermore, it is recommended that the salinity is considered when using the MIR-ATR sensor to quantify the hydrocarbon concentration in natural waters which may contain different amounts of salts.

\subsection{Effect of total petroleum hydrocarbon (TPH)}

Considering that the response mechanism of the MIR-ATR sensor is dictated primarily by partitioning processes it is expected that the extraction of hydrocarbons by the polymer film will depend on the nature and composition of the solution. To evaluate the influence of total petroleum hydrocarbon concentration on analyte quantification and to determine if there is a matrix effect that influences the response, two different types of water samples (saline and pure) were spiked with different amounts of crude oil and measured using the MIR-ATR sensor. The TPH was determined by gas chromatography and it was shown that the aqueous solution contained multiple hydrocarbons (mainly C6 to C36 hydrocarbons). Figure 4 displays the MIR-ATR sensor data versus the GC measured concentration for toluene, $m / p$-xylene and naphthalene, noting that the TPH varied between 1-20 ppm. Generally, the toluene, $m / p$ xylene and naphthalene concentrations determined by the sensor were found to be in reasonable agreement compared to independent GC measurements. Most of the MIR-ATR 
sensor and GC results were within 30\% difference, with the exception of a few toluene and $m / p$-xylene data which varied by more than $50 \%$. Generally, significant differences were observed at low hydrocarbon concentrations and this is mainly attributed to limitations in the MIR-ATR sensor sensitivity/detection limit relative to the GC method. Interestingly, the analytical performance of the sensor did not deviate considerably with increasing TPH, noting that the sensor data was relatively close to the ideal line. The slopes of the sensor versus GC line for the individual hydrocarbon analytes ranged between 0.93 to 1.05 and the correlation coefficients were generally greater than 0.97 (not shown). The linear relationship between the sensor and GC data under a range of different TPHs signifies that the hydrocarbon matrix has a negligible effect on the partition process and hence MIR-ATR sensor sensitivity. This study confirms that the MIR-ATR sensor can accurately quantify certain hydrocarbons in different types of water providing that calibrations are undertaken in solutions and standards of similar salinity/ionic strength.
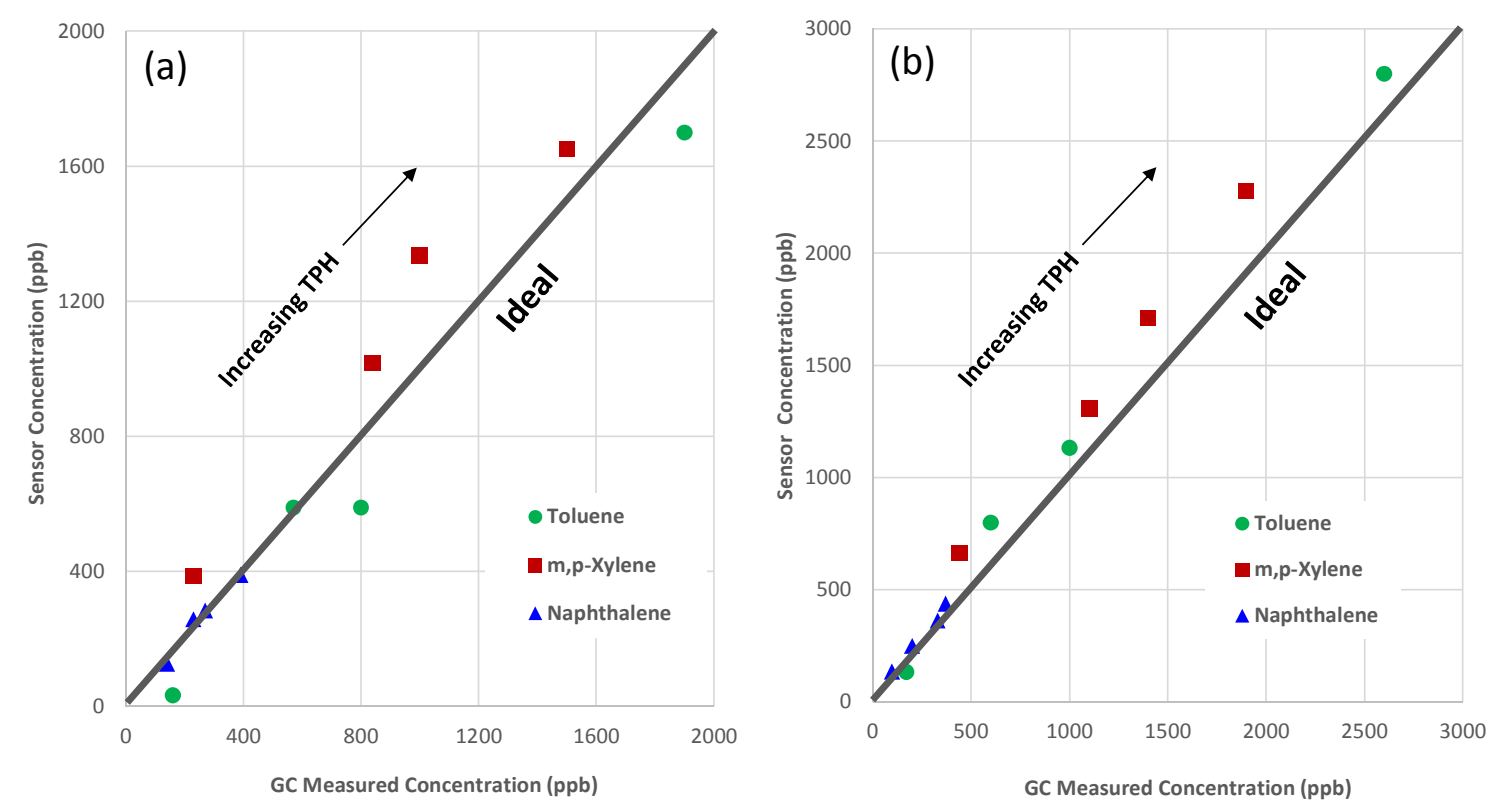

Figure 4. MIR-ATR sensor determined concentration versus gas chromatography for toluene, $m, p$-xylene and naphthalene in (a) saline water (3.5\% salinity) and (b) deionised water as a function of total petroleum hydrocarbon (TPH) concentration. 


\section{Conclusions}

We have shown that the calibration parameters (i.e., response slope) of MIR-ATR sensors depend on the aqueous solution properties (i.e., salinity/ionic strength). The sensitivity of the MIR-ATR sensor was found to be higher in saline solution that comprises different salts when compared to pure water. The sensitivity difference with salinity is consistent with variations in the water-polymer partition coefficient, and suggests that reliable hydrocarbon quantification can be achieved in real environmental samples providing that the sensor is calibrated using appropriate standard solutions and corrected for salinity/ionic strength. Studies were also conducted in a range of oil-water mixtures revealing that the total petroleum hydrocarbons in solution (i.e., at concentrations of 1-20 ppm) does not significantly affect the hydrocarbon-polymer partition process, and hence quantification of the obtained spectral data. More importantly, it was shown that the MIR-ATR sensor provides robust signals in aqueous solutions over extended periods of time, and that degradation of the polyisobutylene film is not a significant issue at ambient temperatures (i.e., $20 \pm 5^{\circ} \mathrm{C}$ ).

\section{Conflicts of Interest}

There are no conflicts of interest to declare.

\section{Acknowledgements}

The authors greatly acknowledge funding provided by CSIRO Energy. We thank the National Geosequestration Laboratory (NGL) for funds to purchase the FTIR spectrometer. RS acknowledges CSIRO for a stipend that enabled them to undertake the present studies. BM acknowledges partial support by the European Union within project SCHeMA (Integrated in Situ Chemical Mapping Probes). Koon-Bay Ho from the National Measurement Institute is thanked for the GC analysis. 


\section{References}

1. S. Mitra, D. G. Kimmel, J. Snyder, K. Scalise, B. D. McGlaughon, M. R. Roman, G. L. Jahn, J. J. Pierson, S. B. Brandt, J. P. Montoya, R. J. Rosenbauer, T. D. Lorenson, F. L. Wong and P. L. Campbell, Geophysical Research Letters, 2012, 39, L01605.

2. U. R. Sumaila, A. M. Cisneros-Montemayor, A. Dyck, L. Huang, W. Cheung, J. Jacquet, K. Kleisner, V. Lam, A. McCrea-Strub, W. Swartz, R. Watson, D. Zeller and D. Pauly, Canadian Journal of Fisheries and Aquatic Sciences, 2012, 69, 499-510.

3. M. V. Storey, B. van der Gaag and B. P. Burns, Water Research, 2011, 45, 741-747.

4. N. S. Chary and A. R. Fernandez-Alba, TrAC - Trends in Analytical Chemistry, 2012, 32, 60-75.

5. B. Pejcic, P. Eadington and A. Ross, Environmental Science \& Technology, 2007, 41, 6333-6342.

6. B. Mizaikoff, Chemical Society Reviews, 2013, 42, 8683-8699.

7. R. Lu, G. Sheng, W. Li, H. Yu, Y. Raichlin, A. Katzir and B. Mizaikoff, Angewandte Chemie - International Edition, 2013, 52, 2265-2268.

8. F. Rauh, M. Schwenk, B. Pejcic, M. Myers, K. B. Ho, L. Stalker and B. Mizaikoff, Talanta, 2014, 130, 527-535.

9. T. Schädle, B. Pejcic and B. Mizaikoff, Analytical Methods, 2016, 8, 756-762.

10. A. Gonzalvez, S. Garrigues, M. de la Guardia and S. Armenta, Analytical Methods, 2011, 3, 43-52.

11. B. Pejcic, M. Myers and A. Ross, Sensors, 2009, 9, 6232-6253.

12. M. Karlowatz, M. Kraft and B. Mizaikoff, Analytical Chemistry, 2004, 76, 2643-2648.

13. K. Flavin, H. Hughes, V. Dobbyn, P. Kirwan, K. Murphy, H. Steiner, B. Mizaikoff and P. McLoughlin, International Journal of Environmental Analytical Chemistry, 2006, 86, 401-415.

14. J. Yang and S. S. Tsai, Analytica Chimica Acta, 2002, 462, 235-244.

15. J. Yang and A. Ramesh, Analyst, 2005, 130, 397-403.

16. B. Pejcic, L. Boyd, M. Myers, A. Ross, Y. Raichlin, A. Katzir, R. Lu and B. Mizaikoff, Organic Geochemistry, 2013, 55, 63-71.

17. F. Regan, F. Walsh and J. Walsh, International Journal of Environmental Analytical Chemistry, 2003, 83, 621-631.

18. A. M. S. Silva, M. F. Pimentel, L. M. Raimundo and Y. M. B. Almeida, Vib. Spectrosc., 2008, 46, 39-44.

19. K. Flavin, H. Hughes and P. McLoughlin, International Journal of Environmental Analytical Chemistry, 2007, 87, 29-42.

20. J. Yang and Y. S. Huang, Applied Spectroscopy, 2000, 54, 202-208.

21. C. Heath, M. Myers and B. Pejcic, Proceedings of IEEE Sensors, 2017, 7808851.

22. P. Heinrich, R. Wyzgol, B. Schrader, A. Hatzilazaru and D. W. Lubbers, Applied Spectroscopy, 1990, 44, 1641-1646.

23. R. Gobel, R. Krska, R. Kellner, R. W. Seitz and S. A. Tomellini, Applied Spectroscopy, 1994, 48, 678-683.

24. B. Pejcic, M. Myers, E. Crooke, A. Ross and M. Baker, Proceedings of IEEE Sensors 2009, 5398158, 299-303.

25. B. Pejcic, M. Myers, N. Ranwala, L. Boyd, M. Baker and A. Ross, Talanta, 2011, 85, 1648-1657.

26. C. Heath, B. Pejcic and M. Myers, New Journal of Chemistry, 2017, 41, 6195-6202.

27. T. Schädle, B. Pejcic, M. Myers and B. Mizaikoff, Analytical Chemistry, 2014, 86, 9512-9517.

28. R. Stach, B. Pejcic, E. Crooke, M. Myers and B. Mizaikoff, Analytical Chemistry, 2015, 87, 12306-12312. 
29. H. Steiner, K. Staubmann, R. Allabashi, N. Fleischmann, A. Katzir, Y. Reichlin and B. Mizaikoff, Water Science and Technology, 2003, 47, 121-126.

30. F. Regan, Instrumentation Science and Technology, 2014, 42, 1-14.

31. R. A. Lyons, J. P. Hassett, A. M. Flach and I. Cabasso, Journal of Applied Polymer Science, 2013, 127, 177-182.

32. F. Smedes, R. W. Geertsma, T. Van Der Zande and K. Booij, Environmental Science and Technology, 2009, 43, 7047-7054.

33. M. T. O. Jonker, S. A. Van Der Heijden, M. Kotte and F. Smedes, Environmental Science and Technology, 2015, 49, 6791-6799.

34. D. R. Kester, I. W. Duedall, D. N. Connors and R. M. Pytkowicz, Limnology and Oceanography, 1967, 12, 176-179.

35. Y. Tsvetnenko and L. Evans, Marine Pollution Bulletin, 2002, 45, 148-156.

36. R. Howley, B. D. MacCraith, K. O'Dwyer, P. Kirwan and P. McLoughlin, Vib. Spectrosc., 2003, 31, 271-278.

37. C. K. Bryant, P. T. LaPuma, G. L. Hook and E. J. Houser, Analytical Chemistry, 2007, 79, 2334-2340.

38. P. McLoughlin, K. Flavin, P. Kirwan, B. Murphy and K. Murphy, Sensors and Actuators B-Chemical, 2005, 107, 170-177.

39. B. Pejcic, E. Crooke, L. Boyd, C. M. Doherty, A. J. Hill, M. B. Myers and C. White, Analytical Chemistry, 2012, 84, 8564-8570.

40. C. Phillips, M. Jakusch, H. Steiner, B. Mizaikoff and A. G. Fedorov, Analytical Chemistry, 2003, 75, 1106-1115.

41. B. Pejcic, E. Crooke, C. M. Doherty, A. J. Hill, M. Myers, X. B. Qi and A. Ross, Analytica Chimica Acta, 2011, 703, 70-79.

42. C. Heath, M. Myers and B. Pejcic, Analytical Chemistry, 2017, 89, 13391-13397.

43. M. Kraft and B. Mizaikoff, International Journal of Environmental Analytical Chemistry, 2000, 78, 367-383.

44. K. M. G. Lima, I. M. Raimundo and M. F. Pimentel, Sensors and Actuators BChemical, 2007, 125, 229-233.

45. J. Yang and M. L. Cheng, Analyst, 2001, 126, 881-886. 

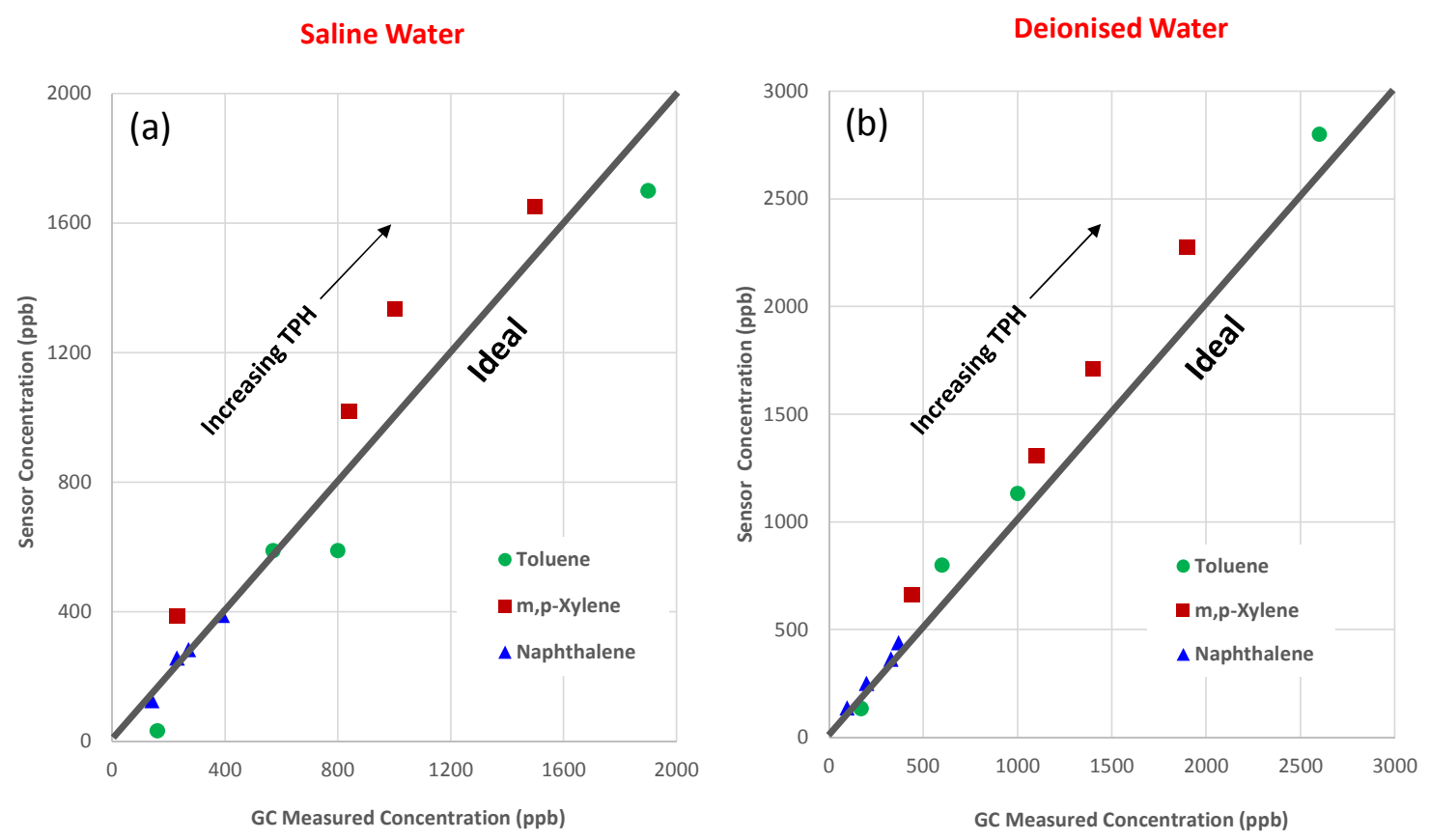

Mid-infrared sensors based on ATR spectroscopy provide a robust and reliable platform for the quantification of organic molecules dissolved in water. 